\title{
A Study on DC Limit Parameters in RCD Operation Using Capacitor
}

\author{
Erwin Sutanto ${ }^{1, *}$, Khusnul AIN $^{1}$, Muhammad AZIZ $^{2}$ and Guillermo ESCRIVÁ-ESCRIVÁ ${ }^{3}$ \\ ${ }^{1}$ Biomedical Engineering, Faculty of Science and Technology, Universitas Airlangga, Surabaya, Indonesia \\ ${ }^{2}$ Institute of Industrial Science, The University of Tokyo, Tokyo, Japan \\ ${ }^{3}$ Institute for Energy Engineering, Universitat Politècnica de València, Spain
}

Received 9 July 2019; Accepted 9 September 2019

\begin{abstract}
Residual-current devices (RCDs) are used to protect humans from electrical shocks. This study explores the DC limit parameters of RCDs using the capacitor. To find out the possible parameters, a common signal from the RCD in AC waveform is analyzed using a digital oscilloscope for reference. This signal is then transformed into a DC signal using a common circuit of a full-bridge rectifier, capacitor, and a voltage regulator. Finally, the signal is characterized using an oscilloscope and multimeter. The result of this work is I-V characteristic of the DC measurement obtained from the RCD. It shows two possible parameters. First is a voltage gap of $1 \mathrm{~V}$ which representing input current with a range from 0.4 to $64.3 \mathrm{~mA}$. Second is an increasing speed of $1.06 \mathrm{~V} / \mathrm{s}$ on average. These parameters could be used to overcome the nuisance trips of common RCDs.
\end{abstract}

Keywords: Residual Current Measurement, Electrical Safety Devices, RCD (Residual Current Device), DC Measurement, Capacitor

\section{Introduction}

A residual-current device $(\mathrm{RCD})$, or similarly ground fault circuit interrupter (GFCI) is a well-known electrical safety device that is used to protect humans from electrical shocks due to leakage current [1]. It deals with a current that flows out of its supposed directions/connections. RCDs can automatically cut off the power connection once a limited over current is detected. Leakage current can occur because of several failures, including failure beyond the human mistakes, broken cables, and even poor conditions of components.

Hence, RCDs are considered important to protect against any possible electrical shocks. A simple approach to maintain electrical safety is by using grounding [2]. Therefore, any leakage current discharged from the device body will be directed to the ground connection. In this case, an individual will be safe from electric shock as it flows out to the ground. However, in the case of ungrounded medical devices, the leakage current could pass through the human body. Moreover, sudden death could also occur if the leakage current flowing through the human body is larger than a certain limit. Under normal conditions, the human body cannot resist the current of above $30 \mathrm{~mA}$ at $220 \mathrm{~V} \mathrm{AC}[3]$.

As a protective device, RCD has very broad application, including general purpose socket-outlets, uninterruptible power supplies, mobile equipments, variable-speed drives, concealed cable in walls, bath/shower room, and agricultural premises. As one of its application, RCDs are attached to medical devices and placed between the device and the

*E-mail address: erwin_sutanto@fst.unair.aci.id

ISSN: 1791-2377 @ 2019 School of Science, IHU. All rights reserved.

doi:10.25103/jestr.124.02 electricity source, preventing any electrical shock to the patient. RCDs will trip the electric current when a specific leakage current is detected. In general, a 30-mA rated current is used as the standard limit value following the IEC standard. In common electrical installation for buildings, TT networks where both RCD and Earth grounding are used [4]. However, although electrical shocks can be avoided with that kind of insulation, some false trips might be also occured. This could be from the effects of harmonics frequency [5], and also from high frequency [6]. Additionally there are also reports of nuisance trip from RCD usage in situations when there are supply voltage disturbances which may due to current surges and being discharged to the ground [7], [8]. The reason for those trip cases may not be detected easily as it happened very quickly and normally no features from common RCDs to track it. RCDs usually only ensures that its current limit is not exceeded.

In general, those methods could be used in a kind of digital $\mathrm{RCD}$. In fact, few of them are already used for the safety calibration of medical devices in hospitals. About the calibration, this should be done frequently. Following several other tests, the leakage current measurement is one of the required tests. The calibration test normally uses a multi-tester tool. The design and construction have been analyzed in [9]. Additionally, a similar kind of digital RCD has been studied also by using micro-controllers (MCUs) such as PIC18F258 [10]. The signal source from the RCD should not be different. However, the following processing blocks are different from each other at these studies.

Following the initial step designing of fast current releasing DC circuit breaker [11], this work considers the practical side of the RCD's characteristic by using common DC measurements. That is by using a low-pass filter was used with regular approaches. It also evaluates the I-V 
characteristics using those common processing blocks in order to obtain a DC signal from the RCD. It can help us to determine the best limit parameters for kind of Digital RCDs by looking at its statistical parameters.One of those parameters could be then optimized using similar methods of PID control scheme [12].It could be usedalso for further analysis, like the estimation of power consumption for typical appliances [13].

\section{Research Method}

Following the electrical shock cases [13], there are two possibilities as shown in Fig. 1. First one is for save condition when the current flows back to the ground. The flow of leakage current is represented as the blue dash line in Fig. 1a. Then, the second one is as shown in Fig. 1b. In this case, the current might flow back to the ground by going through a different path. In a TT network [3], there might be a common ground rod which is used for direct connection to earth economically. A miscalculation on the earthing system could lead to these kinds of risk. In some medical devices, this condition could occur in cases involving leakage current which are connected to patients. Eventually, it could affect the internal organs, as well as the cause of heart failure and paralysis.

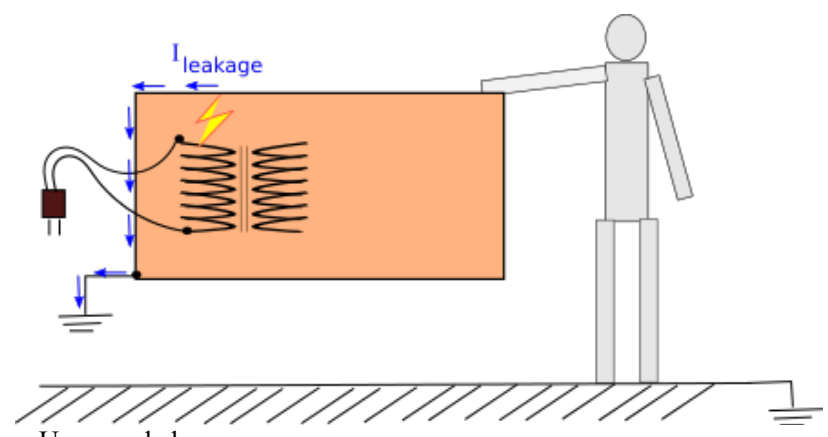

a. Ungrounded

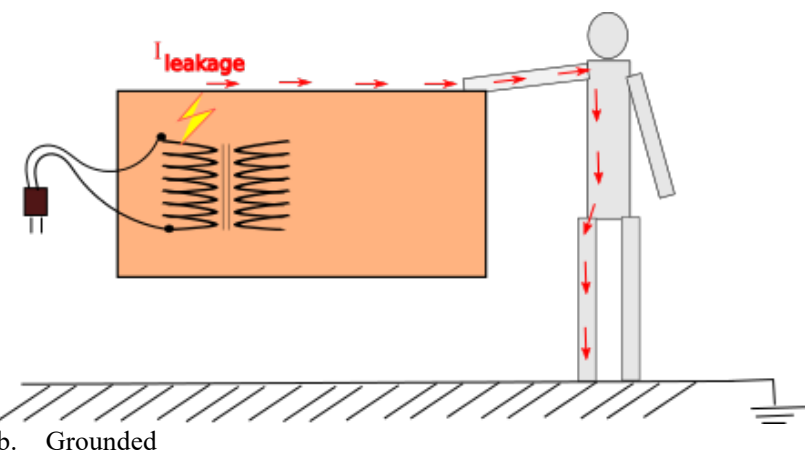

Fig. 1. Macroshock due to ground fault

This section is divided into two parts. First, we focus on two conditions that have been discussed. This could be used to characterize the leakage current which is then read as a residual current. These conditions will also be employed later in the experiment. The second is about equations, which will be useful later when analyzing the signal characteristics.

\subsection{Two Conditions}

A simple test circuit is shown in Fig. 2, and it is used to examine the RCD signals. This test circuit contains an AC power source, phase and neutral cable, a toroid core, and an electrical device as the load. The toroid core itself is at the center of the figure. It has three coils: the incoming coil (blue colored coil), the returning coil (red colored coil), and the sensing coil (yellow colored coil). Two conditions are applied to run the test. These conditions are different in terms of how we connect the toroid core. Points A-B from the blue coils and points C-D from the red coil are linked differently from the AC power source and the load.

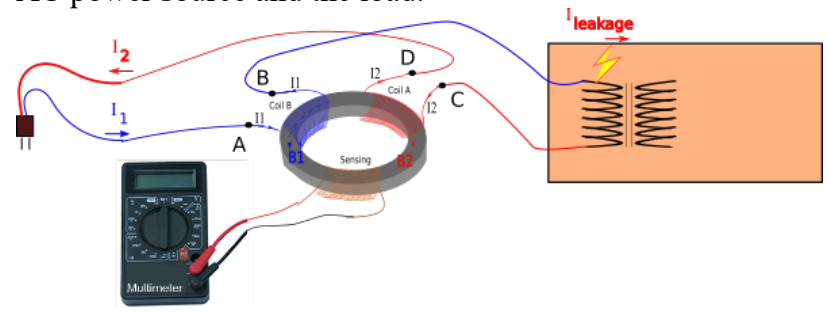

Fig. 2. Simple test circuit of RCD

The first condition is referred to as a "normal condition." In this condition, we applied a normal appliance on the toroid core, and both coils are used. Points A and D of the RCD are connected to the phase cable and neutral cable. Then, points $\mathrm{B}$ and $\mathrm{C}$ are linked to the load. This treatment should generate the same current flowing through the blue coil and red coil. Under ideal circumstances, the current value $I_{1}$ at the blue coil, and current value $I_{2}$ at the red coil could be expressed as in Eq. (1), where they have equal value.

$$
I_{1}=I_{2}
$$

The second condition is referred to as the "leakage current condition" [14]. In this condition, only one of two coils is used, that is, the blue coil. It is made to simulate a leakagecurrent condition as in Fig. 1b. Points A and B of the RCD are still connected to the phase cable and to the load. However, points $\mathrm{C}$ and $\mathrm{D}$ from the red coil are disconnected. This will simulate the figure where all current flows use different paths. It will take a maximum value of $\mathrm{I}_{\text {eakage. }}$ It will reproduce a scenario in which $100 \%$ of the current returns through the grounding path. In general, there will only be a difference between the current that flows through blue and red coils. In that case, the relation between the current value $I_{1}$ in the blue coil and current value $I_{2}$ in the red coil is generally expressed by (2).

$$
I_{1}=I_{2}+I_{\text {leakage }}
$$

\subsection{Measured Signal}

Based on (2), $I_{1}$ is the current flowing through the blue coil, $\mathrm{I}_{2}$ is the current flowing through the red coil, and $\mathrm{I}_{\text {leakage }}$ is the difference between $I_{1}$ and $I_{2}$. The difference in the current values of the two coils will induce an electromagnetic field following Faraday's Law. The electromagnetic induction creates the electric motion force (e.m.f.) voltage, which is readable by the sensing coil. This voltage will be processed with a tripping circuit in normal RCD devices. The change in the magnetic-field value is expressed in equation (3).

$$
\Delta B=B_{1}-B_{2}=\frac{\mu \cdot N}{\theta . r}\left(I_{1}-I_{2}\right)=\frac{\mu \cdot N}{\theta . r} I_{\text {leakage }}
$$

$B_{1}$ and $B_{2}$ are the magnetic fields which is resulted from $\mathrm{I}_{1}$ (incoming current) and $\mathrm{I}_{2}$ (outgoing current) respectively. 
$\mu, \mathrm{N}, \theta, \mathrm{r}$ are the rcd's parameters. $\mu$ is magnetic permeability which depends on the material of the rcd core. $\mathrm{N}$ is the coil's number of turns. $\theta$ is the degree angle of the circular path from RCD core. For a full circle of the toroid, the value will be $2 \pi$. $r$ is the radius of the RCD core, which is together with Omaking the circumference value. Furthermore, with the created magnetic field $\mathrm{B}$, there will be a magnetic flux, $\phi_{\mathrm{B}}$. This can be calculated using A as the cross-sectional area of the toroid. Assume that the cross-sectional area is constant. The rate of change over time, $t$ from magnetic flux rate can be expressed in (4).

$$
\frac{d \varphi_{B}}{d t}=A \frac{d}{d t} B \cos \omega t
$$

Finally, based on Faraday's law $\backslash$ cite $\{k\}$, the magnitude of the e.m.f., $\varepsilon$ in the sensing coil is directly proportional to the magnetic flux rate. It is expressed in (5).

$$
\varepsilon=-\frac{d \varphi_{B}}{d t}
$$

Considering Equations (4) and (5), it could be estimated that the waveform of the $\mathrm{AC}$ voltage is related to the magnetic-field source, $B$. This means that if $B$, as shown in (4), has a frequency at $\omega$, then the sensed AC signal would also have a similar frequency. Then, because B really depends on $\mathrm{I}_{\text {leakage, }}$ it can be said that the frequency would be similar to that of the electric source. Here, a 220-V AC source was used as the electric source.

\section{Results and Analysis}

Using the circuit that has been described in 2.1, the experiment was performed using the two conditions. The first one is to simulate the normal condition, and the second one is for the leakage current condition. Here, the signals were measured using a digital oscilloscope. The first part is for the AC signal. Then, it was continued using the DC measurement's signal. Finally, the I-V characteristics were obtained for different loads.

\subsection{AC signal characteristic}

Before processing the residual current in the digital device, it is necessary to check its original waveform, AC signal first. To do that, the two conditions are used. First, the normal condition requires a normal electricity supply to light the lamp. Then, for the leakage current condition, the returning current $\mathrm{I}_{2}$ is being short-circuited directly to the power source, bypassing the RCD winding. By doing this, we can determine whether the signal is in agreement with the equations. For example, in normal conditions, it is expected that the current measured in the sensing coil will be almost zero. This particular experiment used a 7-W lamp as the load.

For the first condition, the lamp was connected to the blue coils and red coils of the toroid. Then, the sensing coils were connected to the oscilloscope. The current that flows through the blue coils were expected to be the same as the current flows through the red coils. Thus, there should be no current measured in the sensing coils, as follows in Equation (1). The result from the oscilloscope is shown in Fig. 3a. It was slightly different than expected of zero value, although it was a quite small value. The mean voltage was only about $-0.781 \mathrm{mV}$, while the RMS voltage was only $6.25 \mathrm{mV}$. This value showed that there was a small residual current in the circuit instead of following (1). This should be normal as it was under real conditions, or for a non-ideal circuit. It might come from the noise.
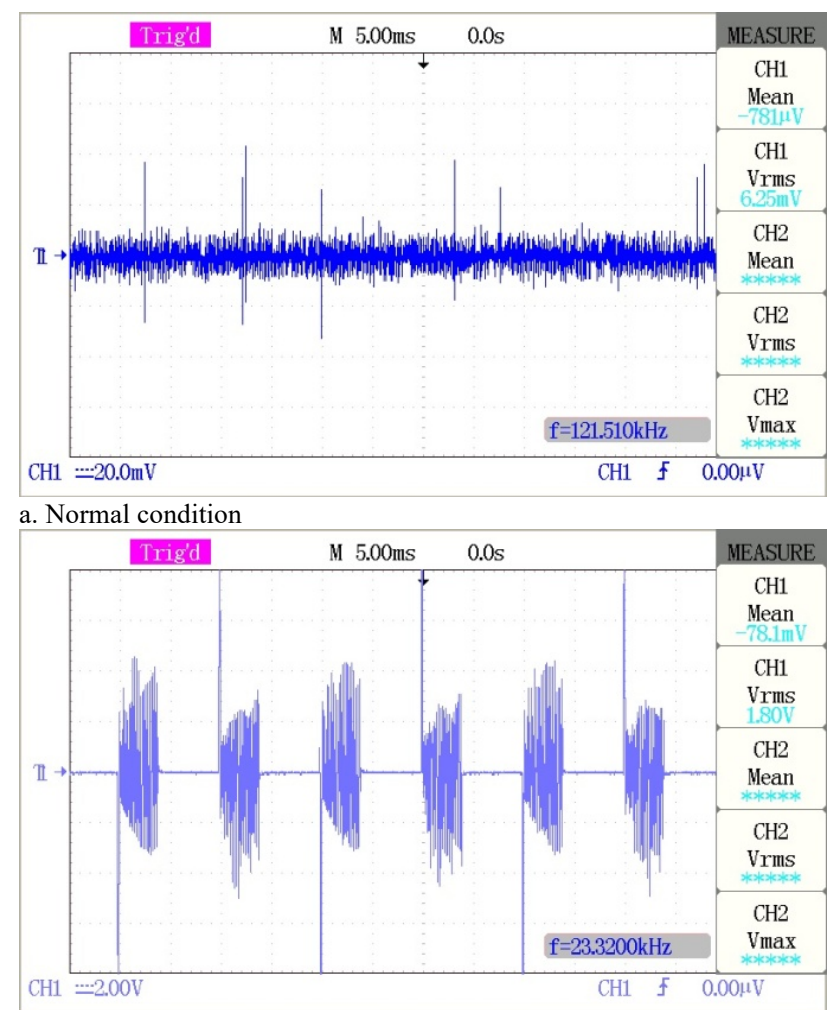

b. Leakage current condition

Fig. 3. AC signal measurement at Sensing Coil

For the second condition, the lamp was connected only to the blue coils, and the red coils are bypassed. Again, the sensing coils were still monitored using the oscilloscope in the normal condition. The measured current in the sensing coils should be in agreement with the following equation (2). Otherwise, because the red coils are bypassed in this case, the value of $\mathrm{I}_{\text {leakage }}$ should be a maximum, as described in 2.1 . In this experiment, the mean voltage was similarly at $-0.781 \mathrm{mV}$, however, the RMS voltage was about $1.80 \mathrm{~V}$. The image from the oscilloscope is shown in Fig. 3b. As expected, the leakage current condition had a higher voltage compared to the normal condition. From the figure, it should be noted that there is a pattern. It could be seen from the peak voltage which is fluctuating over a fixed period. Although the signal waveform did not show fixed values for the peak voltages, or any clear cosine form, the waveform showed a positive peak and negative peak alternately in a similar time period. Measuring that time period, it could be found that it has the same frequency as the input's source, $50 \mathrm{~Hz}$.

\subsection{AC Frequency}

The AC signal data from the digital oscilloscope could be reanalyzed using signal processing software. In this study, we used Octave [16]. Using Octave, it is easy to obtain the frequency spectrum from the $\mathrm{AC}$ signal. We can use a fast Fourier transform (FFT) function to directly convert digital data from the AC signal to its frequency spectrum. To do that, it was first necessary to acquire the raw data from the digital oscilloscope, which is in CSV format. The data file was then loaded into the matrix variable in the Octave environment. After that, the matrix could be easily processed into the $\mathrm{N}$ - 
point discrete Fourier transform (DFT) using the FFT function. In this work, 1024 was used for the N-point.

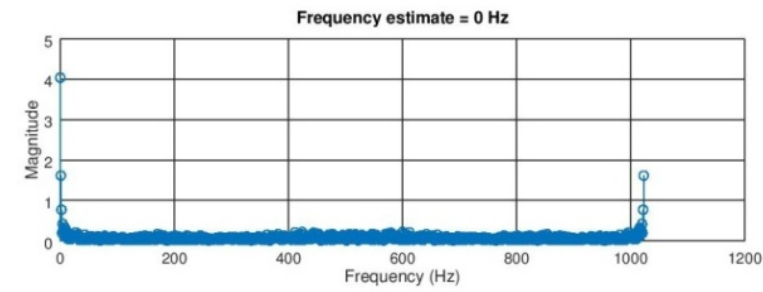

a. Normal

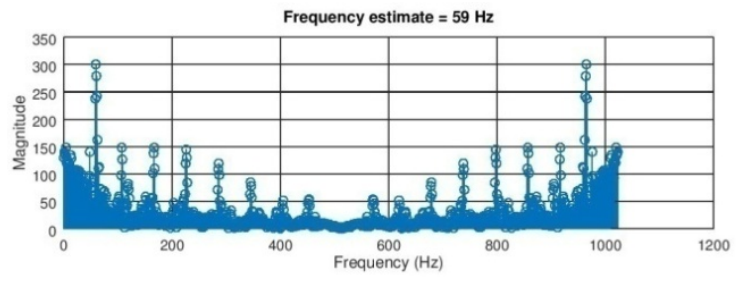

b. Leakage current

Fig. 4. Frequency spectrum of AC signals

The frequency spectrum obtained under normal condition can be seen in Fig. 4a. It can be seen that the maximum value is coming with a frequency of $0 \mathrm{~Hz}$. The figure shows that the maximum value is only reached $4 \mathrm{~V}$. It was a very small number compared to the source, and might follow equation (1). The result was slightly different when compared with one in the leakage current condition. The frequency spectrum on the leakage current condition can be seen in Fig. 4b. The peak data could be observed. It was coming from $59 \mathrm{~Hz}$. It was closed to the source frequency as described in 2.2. This might because of the FFT conversion and the measurement's shift error. To obtain a more accurate frequency, a windowing method from the FFT could be used. However, because it only needed to show the similarity, the differences should be acceptable. Connecting it with the signal waveform in Fig. 3b, the frequency could be obtained also directly from the peak's period as discussed, which fluctuates continually.

\subsection{Measurement}

To obtain a DC voltage over the AC signal, common electronic components were used. The circuit was made from three functional components, namely a rectifier, filter, and regulator. Each component has its own function for a specific task based on its name. The overall circuit is shown in Fig. 5. In this study, only common components are used to measure the DC voltage. The individual components are called blocks as they can be replaced with other components or improved circuit designs in the future to achieve better performance.

The first block is required to convert negative parts of the AC signal into positive values, so it will create absolute values from the AC signal. It utilizes the full-bridge rectifier, which is a common combination of four diodes. The resulting absolute signal will be cut with the threshold voltage of the diode in the lower part of the signal. This is put just after the RCD to read the AC signal directly from the sensing coil, as previously discussed in 3.1 .

After the rectifier, the next block is the filter. A filter was used to increase the stability of the DC signal. Actually, it decreases the DC ripple from the absolute signal of the first block. To realize it, a capacitor was used as a common lowpass filter. This improves the DC value's stability. It is steadier compared with one without a capacitor.

The last processing block is the voltage regulator. For this, a Zener diode was used. Its behavior was affected by its reverse-bias characteristic. It will filter all voltages that exceed its breakdown voltage. In this research, we used a 5$\mathrm{V}$ Zener diode. Thus, it would clip all voltages larger than 5 $\mathrm{V}$. When the voltage is smaller than $5 \mathrm{~V}$, it behaves like a normal diode. This block would make sure that the output is safe for most digital measurement. The measurement is normally in the range of $0-5 \mathrm{~V}$.

With all blocks which have been discussed in the circuit, the original signal will be turned into a DC signal. Although this could be read directly using a microcontroller, in this work, it used only a multimeter and a digital oscilloscope. It is because the purpose was only to find out the characteristic.

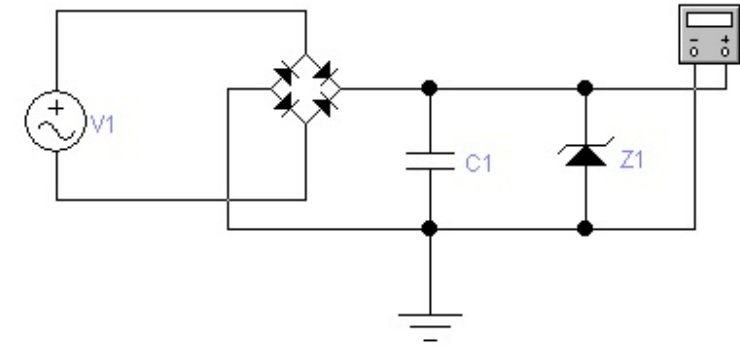

a. Schematic Diagram

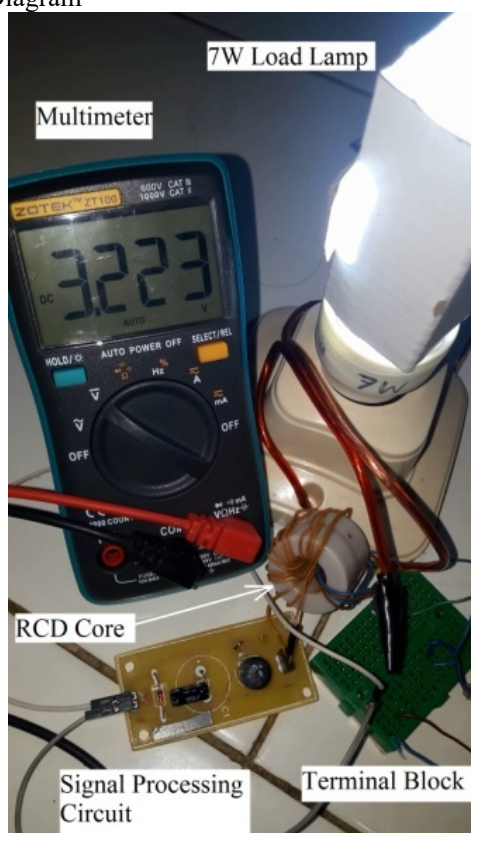

b. Experiment

Fig. 5. Signal processing block diagram

Here, a comparison of the DC signal under two conditions was presented, as in the previous AC signal characteristic. The first one is the normal condition, and the other is the leakage current condition. They are all shown in Fig. 6 . Compared to the AC signal, the waveform of the signal is much different. By analyzing them using an oscilloscope, the waveform has a flat line with a little variation of several $\mathrm{mV}$. These oscilloscope measurements were used only for confirmation of AC to DC transformation using the discussed circuit. The DC measurements were continued later on by using the multimeter. It would be using various loads with different power value on its specification.

Looking at the detail of the normal condition, the DC signal was quite stable. The waveform obtained under the normal condition is shown in Fig. 6a. The stability could be seen from the difference between the RMS value and Mean value. Overall the output voltage is a constant DC value. It was shown as a straight line on the figure. From the figure, it could be seen that RMS value was at $6.25 \mathrm{mV}$, although there 
is a small variation with a peak-to-peak $\mathrm{AC}$ voltage. Meanwhile, the mean value of the signal was only $5.47 \mathrm{mV}$, which is close to the RMS value. The difference is only 0.78 $\mathrm{mV}$. The variation in the result may be due to the measurement's noise. Nonetheless, Fig. 6a already showed that a stable DC voltage is already achieved.
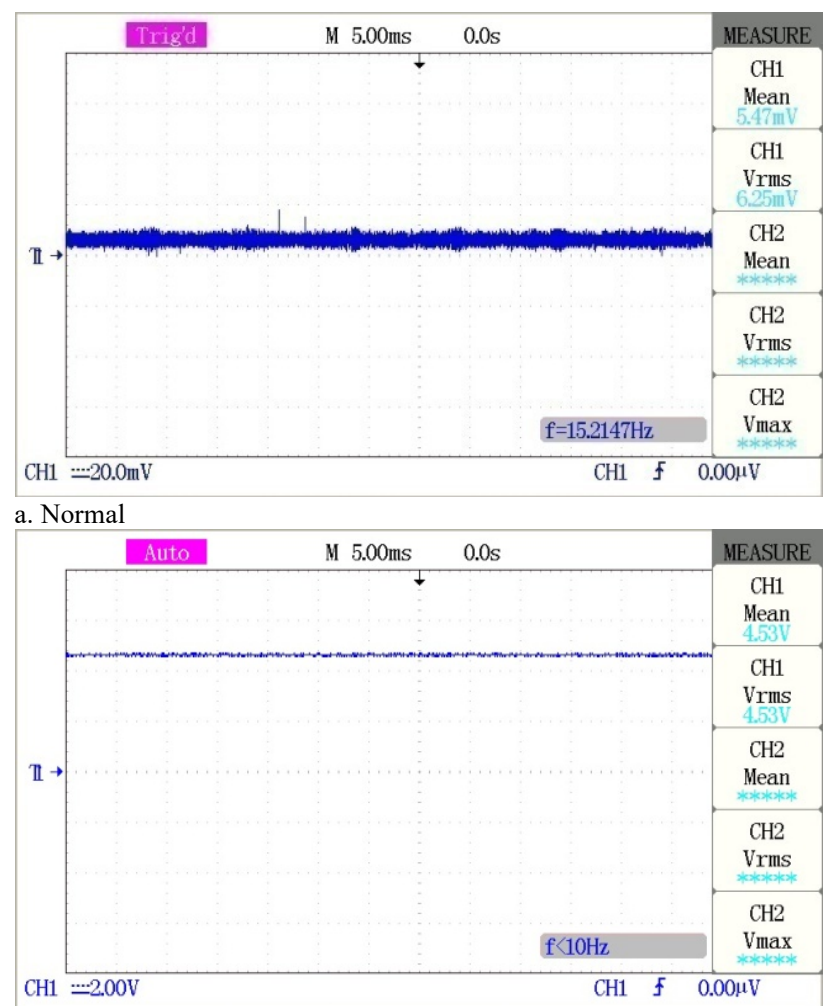

b. Leakage current

Fig. 6. DC measurement

Next, it was possible to explore further possibilities using different load powers. In this work, a compact fluorescent lamp (CFL) was used for the load. The load was varied from 5-15 W. There were seven kinds of load. Table 1 shows the results obtained from the measurement's data. It consists of incoming current, $I_{1}$ and outgoing current $\mathrm{I}_{2}$. Based on those results, the residual current could be calculated using Equation (2) as a replacement for the leakage current. Finally, the DC output could be measured using signal-processing blocks and a multimeter, as shown in Fig. 5. Overall, the data is similar to the load's variation. The mean value of the residual current was $0.4 \mathrm{~mA}$ while the corresponding DC Output from the block was $0.68 \mathrm{~V}$.

Table 1. Comparison of output voltage for various loads in normal conditions

\begin{tabular}{ccccc}
$\begin{array}{c}\text { Power } \\
(\mathbf{W})\end{array}$ & $\mathbf{I}_{\mathbf{1}}(\mathbf{m A} \mathbf{)})$ & $\mathbf{I}_{\mathbf{2}}(\mathbf{m A} \mathbf{)}$ & $\begin{array}{c}\mathbf{I}_{\text {residual }} \\
(\mathbf{m A})\end{array}$ & $\begin{array}{c}\mathbf{D C} \\
\text { Output } \\
\mathbf{( V )}\end{array}$ \\
\hline 5 & 21.0 & 20.6 & 0.4 & 0.68 \\
6.5 & 28.5 & 28.0 & 0.5 & 0.68 \\
7 & 28.8 & 28.1 & 0.6 & 0.67 \\
8 & 34.7 & 34.2 & 0.5 & 0.68 \\
10 & 44.4 & 44.2 & 0.2 & 0.68 \\
13 & 54.8 & 54.6 & 0.2 & 0.67 \\
15 & 64.3 & 64.0 & 0.3 & 0.67 \\
\hline
\end{tabular}

Next, it was possible to explore further possibilities using different load powers. In this work, a compact fluorescent lamp (CFL) was used for the load. The load was varied from
5-15 W. There were seven kinds of load. Table 1 shows the results obtained from the measurement's data. It consists of incoming current, $I_{1}$ and outgoing current $I_{2}$ Based on those results, the residual current could be calculated using Equation (2) as a replacement for the leakage current. Finally, the DC output could be measured using signal-processing blocks and a multimeter, as shown in Fig. 5. Overall, the data is similar to the load's variation. The mean value of the residual current was $0.4 \mathrm{~mA}$ while the corresponding $\mathrm{DC}$ Output from the block was $0.68 \mathrm{~V}$.

Table 2. Comparison of output voltage for various loads in leakage current condition

\begin{tabular}{c|c|c|c}
\hline $\begin{array}{c}\text { Power } \\
(\mathbf{W})\end{array}$ & $\mathbf{I}_{\mathbf{1}}(\mathbf{m A})$ & $\begin{array}{c}\text { DC Initial } \\
\text { Output (V) }\end{array}$ & $\begin{array}{c}\text { Increasing } \\
\text { Speed (V/s) }\end{array}$ \\
\hline 5 & 21.0 & 1.63 & 0.36 \\
6.5 & 28.5 & 2.48 & 1.05 \\
7 & 28.8 & 2.70 & 0.72 \\
8 & 34.7 & 2.56 & 1.60 \\
10 & 44.4 & 2.87 & 3.51 \\
13 & 54.8 & 4.88 & 0.08 \\
15 & 64.3 & 4.90 & 0.07 \\
\hline
\end{tabular}

The DC signal obtained from the leakage current condition has a similar signal shape compared with the one under normal conditions. However, in this state, its output was different. Following the residual current, some measured values were about $5 \mathrm{~V}$. The reason behind it was the Zener diode's voltage. Any voltage exceeds $5 \mathrm{~V}$ would be clipped immediately as described before. A picture from the oscilloscope is shown in Fig. 6b, and shows that V-RMS and the mean value were the same, i.e., $4.53 \mathrm{~V}$.

DC output signal from the leakage current condition was following the normal condition. It was possible to be explored more on how was it with different load's power. The result of the experiment is shown in Table 2. This is a different format compared with Table 1 . Here, there was only an incoming current $\mathrm{I}_{1}$. The outgoing current was bypassed the RCD. Then, the DC output voltage was measured using a similar signal processing circuit, and the multimeter, as shown in Fig. 5. However, in this case, the DC output is not a single low voltage value. It fluctuates rapidly into a maximum voltage defined by the regulator of the Zener diode. All of the outputs end with a DC voltage which was about $5 \mathrm{~V}$. The difference was at the increasing condition. It could be classified based on the initial output value and the speed of its voltage's change. The initial value is the first DC value shown by the multimeter. The rate of change of the voltage is shown by the increasing speed and is calculated from the difference in the increased value over time.

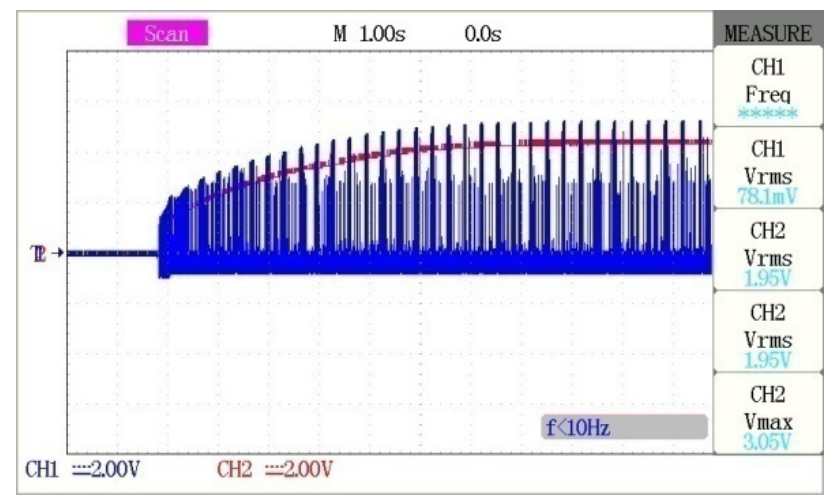

a. Common leakage current condition 
Erwin Sutanto, Khusnul AIN, Muhammad AZIZ and Guillermo ESCRIVÁ-ESCRIVÁ/

Journal of Engineering Science and Technology Review 12 (4) (2019) 7 - 14

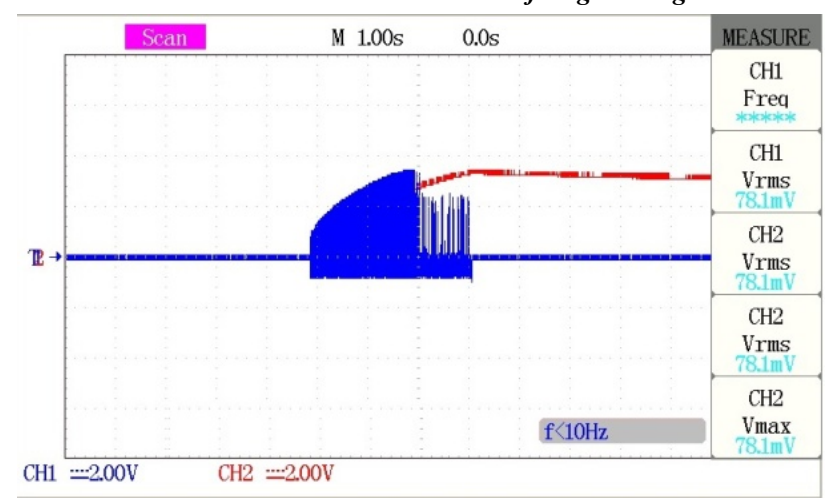

b. Short duration of leakage current

Fig. 7. Measurement response of $7 \mathrm{~W}$ load

The measurement response is shown in Fig. 7. The blue line was using Channel 1 of the oscilloscope (CH1) with the signal coming from the rectifier and capacitor. Meanwhile, the red line was using channel 2 of the oscilloscope $(\mathrm{CH} 2)$ with the signal coming from the output. It showed the speed of output's change in the DC measurement during the leakage current conditions. Fig. 7a showed a common leakage current condition. Meanwhile Fig. $7 \mathrm{~b}$ showed the measurement from a short duration of leakage current. The blue signal was only peaked up for a short time in about $2 \mathrm{~s}$. These data could be seen as the response of the capacitor as a low-pass filter. The filter would slow down the voltage surge at Fig. 7a. Similarly, as indicated by the ac signal waveform before in Fig. 3b, there were high peaks that occur periodically.

The DC output which was shown by red line just followed its input which was shown by blue line. It was increased slowly following the peak after a few seconds depending on the residual current in a similar manner. However, their peak's values were not much difference to each other. It could be seen from both cases in Figure 7. In fact, the output's rms from $\mathrm{CH} 2$ was higher than $\mathrm{CH} 1$. The values of the peaks might depend on the magnitude of the incoming current, $I_{1}$. The peak value can be obtained from the initial DC value. Those values indicate that it can trigger the relay as in common RCD.

Typically, the values increased as the current increased, although it was not linear. For instance, for a load power between 7 and $8 \mathrm{~W}$, there was a decline despite its increased speed, which still increased. Otherwise, for load powers of 13 and $15 \mathrm{~W}$, there was a decline as its speed increased, even though the initial DC output remained high.

Although the capacitor will function as a low-pass capacitor in steady-state response, it will be useful during its transient response. Here, the circuit did not work ideally, as in the circuit in Fig. 5. As discussed and summarized in Table 2 , its DC output peaked at an initial value and then increased with a speed into its maximum value. The value will depend on RCD induced voltage as read at the sensing coil as discussed before at 3.1. Therefore, it could still be modeled by an approximation. Here, it was modeled as a response of the $\mathrm{R}-\mathrm{C}$ circuit's transient response. The resistance itself could be due to several conditions, e.g., the sensing coil's wire or the cable.

$$
V_{o}=V_{i}\left(1-e^{-t / \tau}\right)=5\left(1-e^{-0.72 t}\right)
$$

The increasing response could be simulated as the response of the time constant, $\tau$. It followed the common capacitor's characteristic with $\mathrm{V}_{\mathrm{o}}$ as output voltage and $\mathrm{V}_{\mathrm{i}}$ as the input voltage. For a particular experiment, a 7-W load could be used as in (6). This was with an input voltage of $5 \mathrm{~V}$ and an increasing speed of $0.72 \mathrm{~V} / \mathrm{s}$. This obtained characteristic will be very useful later when identifying between two conditions. It will be practical also for determining the limit parameter for RCD's operation.

\subsection{I-V Characteristic}

By combining both normal conditions in Table 1 and the leakage current condition in Table 2, it is possible to obtain a wide range of voltages for different current values. As shown in Table 1, the data from the normal condition show a low DC output voltage with a narrow range between 0.67 and $0.68 \mathrm{~V}$, as shown in Fig. 8 with a blue marker. The blue markers are looked like a big blue dot which is closed to the vertical axis. Meanwhile, the initial DC outputs from the leakage current condition specified above in Table 2 have a wide range of voltages from 1.63 to $4.90 \mathrm{~V}$. These are shown in the same figure with a small red square. The data is spread from the middle to the upper right corner of the graph. From this figure, it could also be seen that there is a gap between the normal condition and the leakage current condition, which was almost $1 \mathrm{~V}$ on its DC output. Finally, from the overall DC measurement data, it could still be fitted in a linear trend line with $\mathrm{R}^{2}$ around 0.96 . This is shown with a gradient of 0.065 $(\mathrm{V} / \mathrm{mA})$, and a zero adjustment around $0.596 \mathrm{~V}$.

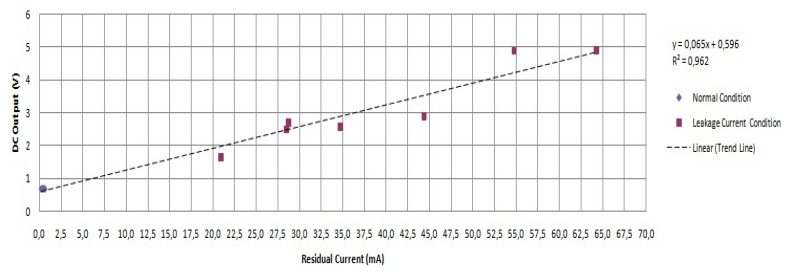

Fig. 8. Dispersion of DC measurements from RCD

The most obvious feature observed in the figure is the different behaviors of the two groups of data. In normal conditions, they appeared to be very close to one value. Conversely, in the leakage current condition, the data were spread over a wide voltage range. This difference was presented in detail in Table 3. The table showed that in the normal condition, the median and mean had the same value of $0.68 \mathrm{~V}$, and there was a very small standard deviation of 0.01 $\mathrm{V}$. In the leakage current condition, the median and mean values were 2.7 and $3.15 \mathrm{~V}$ respectively. In addition, the standard deviation was $1.25 \mathrm{~V}$. Furthermore, there was a difference in how the capacitor is charged for both conditions. In the first group, it appeared that there was no capacitor charge, while for the second group, the capacitor charge rapidly fluctuated. It was shown in the speed column. Its standard deviation was $1.21 \mathrm{~V} / \mathrm{s}$, and the mean value was 1.06 $\mathrm{V} / \mathrm{s}$.

This data could be justified with one more parameter to see its homogeneity. This will quantize the described visual approach earlier. The parameter is the coefficient of variation (CV). This could be calculated using a known formula in (7) with $\sigma$ as the standard deviation and $\mu$ as the mean value. It could be obtained that the dispersion from the normal condition is only $1.5 \%$. Meanwhile from leakage condition, its DC output has a value of $39.7 \%$. Henceforth it is not surprising if the common RCD has a certain limit value because the values in normal condition were almost similar.

$$
C v=\frac{\sigma}{\mu} \times 100 \%
$$


Table 3. Overall dispersion of DC measurement

\begin{tabular}{c|c|c|c}
\hline Condition & Parameter & $\begin{array}{c}\text { DC } \\
\text { Output (V) }\end{array}$ & Speed (V/s) \\
\hline \multirow{5}{*}{ Normal } & Minimum & 0.67 & - \\
& Median & 0.68 & - \\
& Maximum & 0.68 & - \\
& Mean & 0.68 & - \\
& Std. & 0.01 & - \\
& Deviation & & \\
\hline \multirow{3}{*}{ Leakage } & Minimum & 1.63 & 0.07 \\
Current & Median & 2.70 & 0.72 \\
& Maximum & 4.90 & 3.51 \\
& Mean & 3.15 & 1.06 \\
& Std. & 1.25 & 1.21 \\
& Deviation & & \\
\hline
\end{tabular}

The behavior may be simply used in common RCDs by utilizing its current value and choosing one trip point. Normally, it would be limited to $30 \mathrm{~mA}$. It should also be employed as the safety standard for leakage current [3] and is recommended for common residential usage. However, there is a report about nuisance trips [7]. By rule, a $30 \mathrm{~mA}$ RCD should not trip with currents lower than $15 \mathrm{~mA}$ and should have tripped for currents higher than $30 \mathrm{~mA}$. However, in a standard RCD, it could trip with rate currents around 23-24$25 \mathrm{~mA}$ which have been simulated also with specific conditions [8]. That particular limit could be improvised using this presented data for digital RCDs. In this kind of RCDs, besides performing DC measurements, it might be used for further calculation before it really should be tripped at a certain limit. The signal processing block may vary, although the source might be similar as in [9], [10]. The output signal could be equally in DC voltage. Considering the I-V characteristic, the gap may be around $1 \mathrm{~V}$. The trip point would depend not only on the minimum voltage of the leakage current at $1.63 \mathrm{~V}$ for example, but may also employ the increasing speed, as in the capacitor charge, which has an average of $1.06 \mathrm{~V} / \mathrm{s}$. For the increasing speed, the coefficient of variation is about $114 \%$. It was not showing a good homogeneity. However, it was existed here in the leakage current condition. Consequently, it might be used.

As a common RCD, it could be used for two basic safety applications. First, it would prevent currents path through the users as indirect contact safety. Second, it might also open the circuit and made the protection for the users as direct contact safety. Additionally, it could be used to protect facilities because it will open the circuit when there was a leakage current by a fault in the insulation of the conductors or equipments.

Following the additional case, a microcontroller could be planted with this RCD. If that microcontroller or any other digital controller was used with memory, then the tracing voltage could also be recorded. Then, the real residual current value of $30 \mathrm{~mA}$ could be justified. Besides, it could be seen that there was initial DC value which in between 2 and $3 \mathrm{~V}$. If the same linear trend line was used for the standard current limit, it would have $2.55 \mathrm{~V}$ as the limit. In this kind of conditions were in normal or any conditional operation, any unwanted trips could happen in normal RCDs. Thus, the DC measurement should not be justified as the only parameter for the limit. It might be necessary to employ the increasing speed as one of the parameters also. By obtaining all DC values under two conditions, it should be possible to provide both the electrical safety and leakage current tracks. The electrical safety can be realized by utilizing the two described parameters. The first parameter is the DC output limit, which will filter out the average leakage current condition. The second parameter is the increasing speed limit, which will help with the identification of the leakage conditions of loads with high power. Finally, the leakage current tracks would be available during the normal condition, such as the low voltage with a limited voltage change. Here, from the leakage current tracks, the detection of odd behavior could be optimized. Accordingly, a further study should be carried out to demonstrate the feasibility and its effectiveness on detection of nuisance trips.

\section{Conclusion}

The dispersion from overall DC measurements of the RCD shows that the data obtained from the two conditions were fitted in a linear trend line with a value of $\mathrm{R}^{2}$ close to one, which is 0.96 . There was a gap also between normal usage and when there is a leakage current. It was about $1 \mathrm{~V}$. In this DC measurement of leakage current conditions, some initial DC outputs were found also higher than the mapped voltage of standard current limit using the same trend line. The detection of these leakage current conditions could be optimized by utilizing the increasing speed of the capacitor charge. By assuming if nuisance trips could have specific increasing speed, its detection would be also possible if one plants this RCD with microcontroller. One of its applications could be kind of tracing tool, in addition to the safety one. However, a deeper study should be done in order to avoid any incidences of unwanted trips while keep maintaining safety as a priority.

\section{Acknowledgements}

The work and publications are supported by the Faculty of Science and Technology, UniversitasAirlangga with a Young Lecturer's Research Fund Program. This article would not have been completed without the support of all the team members. Many thanks for all of the team members, as well as other colleagues for their suggestions and indirect assistance.

This is an Open Access article distributed under the terms of the Creative Commons Attribution License

\section{References}

[1] J. Webster, Medical instrumentation: application and design. John Wiley \& Sons, 2009.

[2] C.-H. Lee and S.-D. Lin, "Safety assessment of ac earthing systems in a dc traction-supply substation," IEE Proceedings-Electric Power Applications, vol. 152, no. 4, pp. 885-893, 2005.
[3] I. E. Commission et al., "Iec 60364-4-41low voltage electrical installationspart 4-41: Protection for safetyprotection against electric shock,'International Standard, 2005.

[4] X. Zhang, H. Cao, C. Zhao, M. Xu, T. Li, D. Ye, J. Zhang, J. Zhu, Y. Yue, and Y. Song, "Discussion on leakage protection in distribution network," in 2018 International Conference on 
Erwin Sutanto, Khusnul AIN, Muhammad AZIZ and Guillermo ESCRIVÁ-ESCRIVA'

Journal of Engineering Science and Technology Review 12 (4) (2019) 7 - 14

Advances in Social Sciences and Sustainable Development (ASSSD 2018). Atlantis Press, 2018.

[5] T. Lee and T. Chan, "The effects of harmonics on the operational characteristics of residual-current circuit breakers," in Energy Management and Power Delivery, 1995. Proceedings of EMPD'95., 1995 International Conference on, vol. 2. IEEE, 1995, pp. 548-553.

[6] F. Freschi, "High-frequency behavior of residual current devices," IEEE Transactions on Power Delivery, vol. 27, no. 3, pp. 16291635, 2012.

[7] C. Roldán-Porta, G. Escrivá-Escrivá, F.-J. Cárcel-Carrasco, andC. Roldán-Blay, "Nuisance tripping of residual current circuit breakers: A practical case," Electric Power Systems Research, vol. 106, pp. 180-187, 2014.

[8] G. Escriva-Escriva, C. R. Porta, and E. C. de Jong, "Nuisance tripping of residual current circuit breakers in circuits supplying electronic loads,'Electric Power Systems Research, vol. 131, pp. 139-146, 2016.

[9] T. Kaewgun, K. Yantapan, and P. Anupongongarch, "Multi-function electrical safety tester for medical equipment application," in Biomedical Engineering (BME-HUST), International Conference on. IEEE, 2016, pp. 57-60.
[10]G. Rongyan and Z. Honghui, "Study on the residual current protection device technology," Open Electrical \& Electronic Engineering Journal, vol. 8, pp. 404-411, 2014.

[11]A. Mokhberdoran, A. Carvalho, N. Silva, H. Leite, and A. Carrapatoso, "Design and implementation of fast current releasing dc circuit breaker," Electric Power Systems Research, vol. 151, pp. 218-232, 2017.

[12]A. Nassiopoulos, "An embedded pid temperature control scheme with application in a medical microwave radiometer," Journal of Engineering Science and Technology Review, vol. 9, no. 4, pp. 56-60, 2016.

[13]P. A.-D.-V. Raj, M. Sudhakaran, P. P.-D.-A. Raj et al., "Estimation of standby power consumption for typical appliances," Journal of Engineering Science and Technology Review, vol. 2, no. 1, pp. 71-75, 2009.

[14]E. Sutanto, F. Chandra, E. Gonnelli et al., "Residual current measurement using helmholtz coil configuration with different current flow." International Journal of Electrical \& Computer Engineering (2088-8708), vol. 8, no. 3, 2018.

[15] W. Lewin and B. John, "Physics ii electricity and magnetism," 2007.

[16] M. Murphy, "Octave: A free, high-level language for mathematics," Linux journal, vol. 1997, no. 39es, p. 8, 1997. 